\title{
EFFECTS OF MATERIAL PROPERTIES ON RESONANCE FREQUENCY OF A CESR-III TYPE 500 MHZ SRF CAVITY
}

\author{
M.C. Lin*, Ch. Wang, L.H. Chang, G.H. Luo, NSRRC, Taiwan \\ M.K. Yeh, National Tsing Hua University, Taiwan
}

\begin{abstract}
A simulation that links the calculation of mechanical structure and of radio frequency electromagnetic field with the finite element code ANSYS ${ }^{\circledR}$ is employed in this study. Both efficiency and accuracy of the calculation are thus improved over those of the conventional computation that builds separate models with different codes for each computed domain because information related can be internally transferred between the different computation domains. The resonance frequency changes associated with mechanical properties of niobium are examined. The computed results indicate that the coefficient of thermal expansion significantly affects the shift in resonance frequency that occurs when the cavity is cooled from room temperature to liquid helium temperature. Young's modulus and Poisson's ratio have minor effects.
\end{abstract}

\section{INTRODUCTION}

As a major upgrade, one CESR-III $500 \mathrm{MHz}$ SRF module will be installed in the electron storage ring at the National Synchrotron Radiation Research Center, Taiwan, to double the stored electron beam and consequently the synchrotron light density, as well as to eliminate the higher order mode (HOM) effects [1]. This SRF cavity is thus examined herein.

The shell-like cavity inside this SRF module is made of $3 \mathrm{~mm}$-thick bulk niobium sheets. This cavity is immersed in liquid helium to be maintained in a superconducting state. The saturation temperature of $\mathrm{He}^{4}$ at $1 \mathrm{~atm}$ (101.33 $\mathrm{kPa}$ ) is $4.22 \mathrm{~K}$. The mechanical properties of niobium at room temperature and at cryogenic temperature have been tested $[2,3]$ to some extent, and found to depend very strongly on purity, manufacture, and the post treatment, like heat treatment. However, the cavity structure shrinks as it is cooled down to cryogenic temperature by liquid helium. Consequently the resonance frequency of the accelerating mode, the $\mathrm{TM}_{010}$-like mode, raises primarily because it is inversely proportional to the radius of the cavity. Besides thermal contraction, the SRF cavity is also loaded by external pressure, since it has an ultra-high vacuum inside while the liquid helium applies external pressure. Hence the resonance frequency shift is a total effect of cooling and pressuring.

A coupled domain simulation can predict the RF characteristics after cooling. The commercially available finite element code ANSYS $^{\circledR}$ has provided the capability to link the high-frequency electromagnetic computation with structure computation. Such a linked simulation is

chyuan@srrc.gov.tw efficient because compatible models of these two domains can be established and integrated using this software. The related calculated data can then be transferred since the meshes of models employed at these two domains are compatible with each other.

The technique is applied herein to predict the RF characteristics of the CESR-III $500 \mathrm{MHz}$ SRF cavity when cooled and externally loaded. This study focuses mainly on the variation of the electromagnetic resonance frequency. For simplicity, the RF input coupler structure is not considered. The effects of material properties on the electromagnetic resonance frequency are examined. At first, the deflection is determined using a shell structure model. The coordinates of all the surface nodes in the electromagnetic model are updated with the displacements of corresponding shell structure nodes. Then the electromagnetic characteristics of the deflected cavity are computed.

\section{NUMERICAL MODELING}

In calculating the electromagnetic resonance frequency using ANSYS $^{\mathbb{R}}$, a good mesh has been demonstrated to reduce the numerical error in the calculated resonance frequency of a $500 \mathrm{MHz}$ cylindrical pillbox cavity to within $0.1 \%$ of theoretical result [4]. This fact helps the arrangement of the mesh herein. The cavity wall is modeled using shell elements Shell93 for mechanical behavior analysis because it is curved and its thickness is much less than the main dimensions of the cavity. And the brick element HF120 is used in electromagnetic computations because it is appropriate for modeling solids with curved surfaces. The mapped mesh established using the commercial code MSC.PATRAN allows full control of its size and shape.

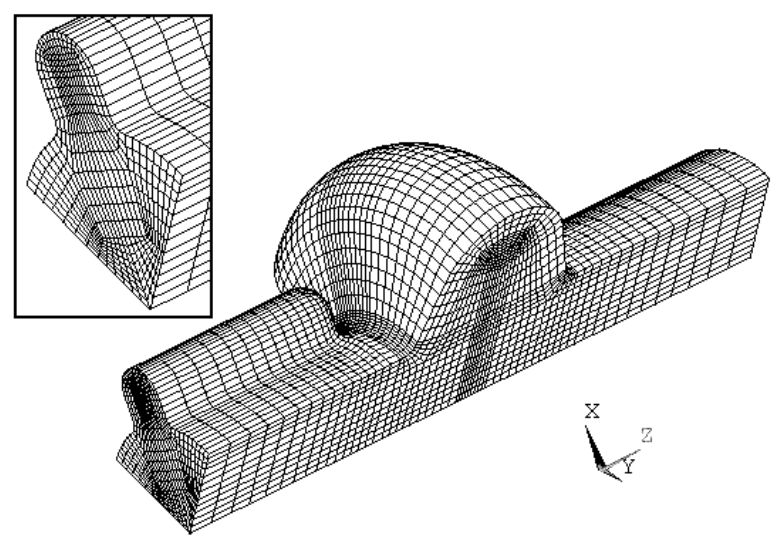

Figure 1: FEM model of the CESR-III $500 \mathrm{MHz}$ SRF cavity. 
Only one quarter of the cavity was modeled because both the structure and the interested electromagnetic fields are symmetric. Figure 1 shows the mesh of the FEM model. This model is comprised of 29474 HF120 elements, 2422 SHELL93 elements, and 126170 nodes. The meshes used in structural and electromagnetic computations are mutually compatible and allow computing information to be transferred between these two computation domains. Both the calculation efficiency and accuracy are thus improved.

In calculating the deflection due to cooling and pressuring, the assigned nodal displacements are the boundary conditions of the fixed end at the round beam tube (RBT) and symmetrical edges at the longitudinal boundaries of the shell model. Additionally, all the nodes at the flute beam tube (FBT) end are constrained to yield the same longitudinal displacement. The applied forces are the pressures on the inner and outer surfaces of the cavity wall and the temperature change.

In calculating the electromagnetic resonance frequency, only the first order transverse magnetic mode, the $\mathrm{TM}_{010^{-}}$ like mode, is considered. Thus, the boundary condition of $A_{X}=0$ is implied at cavity surfaces and both RBT and FBT ends, where $A_{X}$ is the covariant components of the electric field

\section{COMPUTED RESULTS}

During cooling from room temperature to $4.22 \mathrm{~K}$, the SRF cavity is loaded with a temperature change and a pressure difference, so the material properties of interest herein are the coefficient of thermal expansion, Young's modulus and Poisson's ratio. At room temperature, Young's modulus of niobium is $105 \mathrm{GPa}$; its Poisson's ratio is 0.38 , and the coefficient of thermal expansion is $7 \times 10^{-6} \mathrm{~K}^{-1}$. With all these material constants applied and internal vacuum and external pressure of $1 \mathrm{~atm}$, the computed resonance frequency is $500.559 \mathrm{MHz}$ for a CESR-III type $500 \mathrm{MHz}$ SRF cavity at $300 \mathrm{~K}$.

At cold, only the equivalent state is interested herein. From the available data $[2,3]$, a coefficient of thermal expansion of $4.94 \times 10^{-6} \mathrm{~K}^{-1}$; Young's modulus of 125 $\mathrm{GPa}$, and Poisson's ratio of 0.38 were selected as the default niobium properties for numerical computation at $4.22 \mathrm{~K}$ in this work, where no other values are specified.

\section{Coefficient of Thermal Expansion}

The coefficient of thermal expansion is an important property when the temperature change is not negligible. This coefficient declines as the temperature falls for most materials. The equivalent coefficient of thermal expansion is determined as the total thermal contraction ratio over the entire range of temperature change considered. This definition simplifies the numerical computation to avoid incremental computation. The equivalent coefficient is expected to strongly affect both the deflection and the resonant frequency of the SRF cavity.

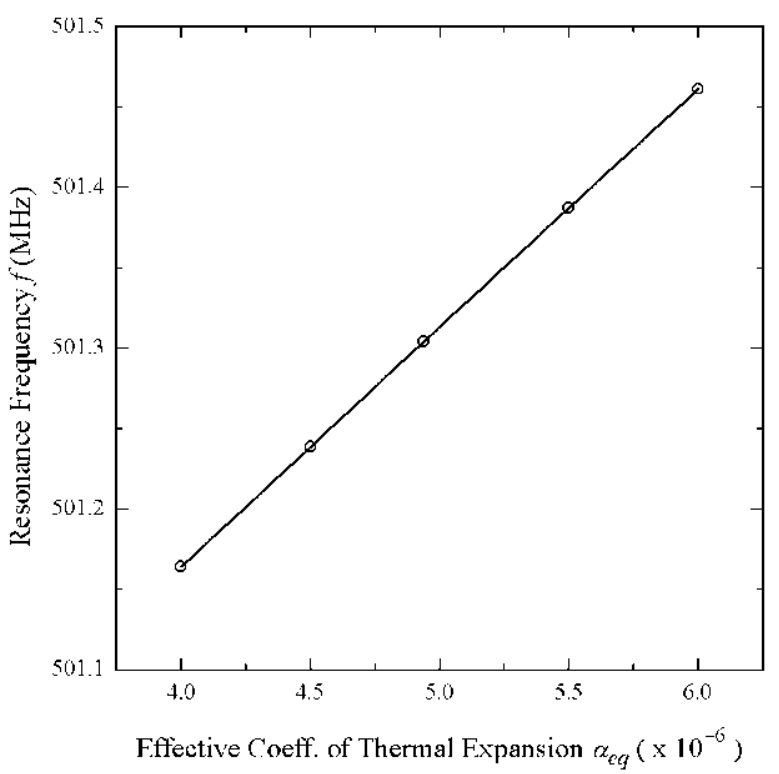

Figure 2: Variation of computed resonance frequency of a CESR-III $500 \mathrm{MHz}$ SRF cavity with various equivalent coefficients of thermal expansion at $4.22 \mathrm{~K}$.

With an internal vacuum and an external pressure $101.33 \mathrm{kPa}$, Fig. 2 shows that the resonance frequency of the CESR-III 500-MHz SRF cavity increases linearly from $501.164 \mathrm{MHz}$ to $501.461 \mathrm{MHz}$ as the equivalent coefficient of thermal expansion increases from $4.0 \times 10^{-6}$ $\mathrm{K}^{-1}$ to $6.0 \times 10^{-6} \mathrm{~K}^{-1}$. A cavity with a higher coefficient of thermal expansion shrinks more when cooled down, and thus has a higher resonance frequency. The variation of computed resonance frequency is approximately $+/-150$ $\mathrm{kHz}$ given $\mathrm{a}+/-20 \%$ variation in the equivalent coefficient of thermal expansion of around $5.0 \times 10^{-6} \mathrm{~K}^{-1}$. Accordingly precise data on the thermal contraction of cavity material at cryogenic temperatures can improve the prediction of the cavity's resonant frequency at a cryogenic temperature.

\section{Young's Modulus}

Briefly, a higher Young's modulus implies greater structural stiffness and, thus, a smaller deflection of a structure under external loading. According to the experiment of Rao and Kneisel [1], Young's modulus of a high-purity niobium sheet is about $125 \mathrm{GPa}$ at $4.2 \mathrm{~K}$. They also stated that the measured Young's modulus of each niobium sheet from different suppliers or undergone different heat treatments varies widely.

Figure 3 plots the variations in the resonance frequency of the CESR-III $500 \mathrm{MHz}$ SRF cavities with various Young's modului given an internal vacuum and an external pressure of $101.33 \mathrm{kPa}$. The cavity's resonance frequency is shown to increase with the Young's modulus at both $300 \mathrm{~K}$ and $4.22 \mathrm{~K}$. This indicates a higher Young's modulus implies greater structural stiffness and thus a smaller deflection and frequency shift. However, 


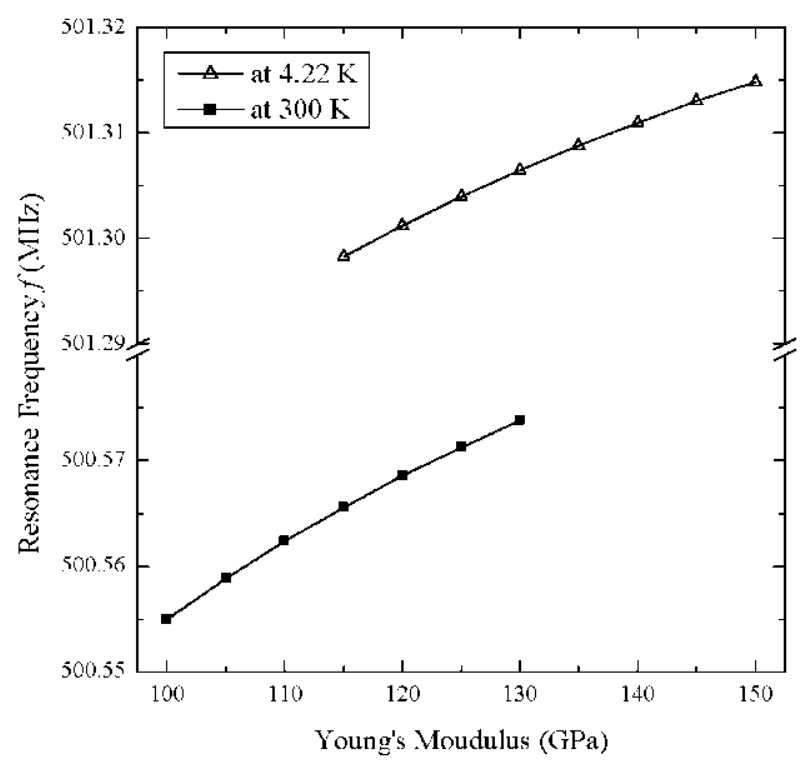

Figure 3: Variation of computed resonance frequency of a CESR-III $500 \mathrm{MHz}$ SRF cavity with various Young's modulus.

over the computed range of Young's modulus, the frequency variation is below $20 \mathrm{kHz}$ at both $300 \mathrm{~K}$ and $4.22 \mathrm{~K}$

\section{Poisson's Ratio}

Poisson's ratio is defined as the negative of the ratio of lateral strain to the stretched longitudinal strain. According to the theory of elastic mechanics, Poisson's ratio is between 0 and 0.5 . Figure 4 shows the variation of the computed resonance frequency of the CESR-III $500 \mathrm{MHz}$ SRF cavity at $4.22 \mathrm{~K}$, under loading conditions of an internal vacuum and an external pressure of 101.33 $\mathrm{kPa}$, as Poisson's ratio changes from 0.1 to 0.5 . Although the resonance frequency decreases as Poisson's ratio increases, the total frequency shift is below $4 \mathrm{kHz}$ in the computed range of Poisson's ratio, as shown in Fig. 4. Thus Poisson's ratio is a minor factor in determining the resonance frequency.

\section{CONCLUSIONS}

A structure-RF coupled computation process with single code is herein used to predict the characteristics of the CESR-III $500 \mathrm{MHz}$ SRF cavity cooled down to liquid helium temperature. The mechanical properties of niobium are examined to find their effects on the resonance frequency of $\mathrm{TM}_{010}$-like mode. According to the computed result, the electromagnetic resonance frequency increases when the SRF cavity is immersed in a liquid helium bath, due to thermal contraction of the cavity. The coefficient of thermal expansion strongly

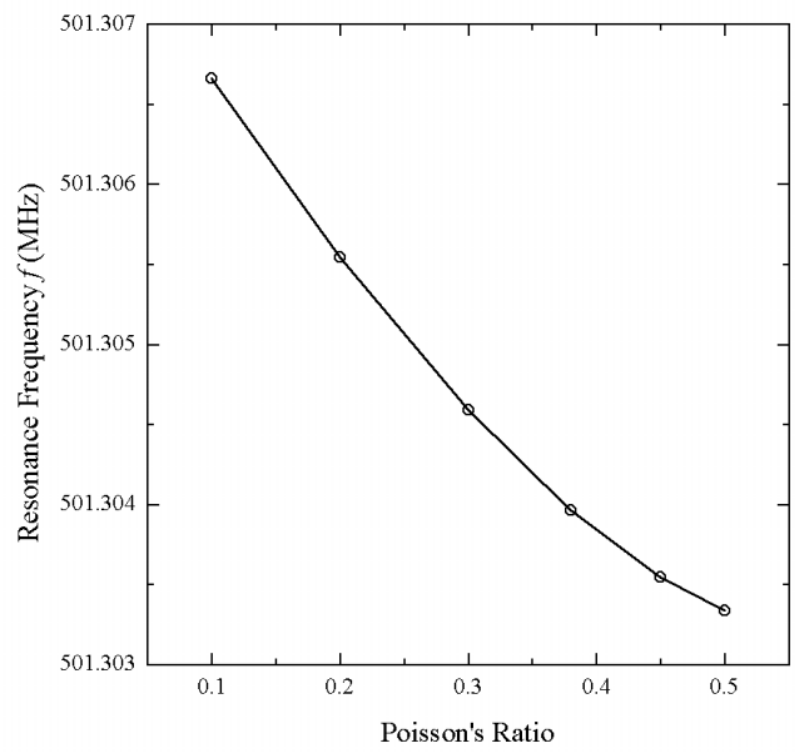

Figure 4: Variation of computed resonance frequency of a CESR-III $500 \mathrm{MHz}$ SRF cavity with various Poisson's ratio at $4.22 \mathrm{~K}$.

affects the shift in resonance frequency when the cavity is cooled from room temperature to liquid helium temperature, whereas Young's modulus and Poisson's ratio have minor effects.

\section{ACKNOWLEDGEMENT}

The authors would like to thank the National Science Council of Taiwan, Republic of China, for partially supporting this research under Contract No. NSC 902213-E-213-001.

\section{REFERENCES}

[1] Ch. Wang, et al., "Superconducting RF Project at the Synchrotron Radiation Research Center," Proceedings of the 10th Workshop on RF Superconductivity, Tsukuba, Japan, 2001, paper ID TL010.

[2] M. G. Rao and P. Kneisel, "Mechanical Properties of High RRR Niobium," Advances in Cryogenic Engineering, 1994, Vol. 40, pp. 1383-1390.

[3] K. Ishio, et al., "Fracture Toughness and Mechanical Properties of Pure Niobium and Welded Joints for Superconducting Cavities at 4K," Proceedings of the 9th Workshop on RF Superconductivity, Santa Fe, New Mexico, USA, 1999, paper ID TUP042.

[4] M. C. Lin, et al., "A Coupled-Field Analysis on RF Cavity," Proceedings of the 2001 Particle Accelerator Conference, 2001, pp. 1207-1209. 